\title{
Análise numérica da flambagem térmica em placas finas de aço
}

\author{
Numerical analysis of the thermal buckling in thin steel plates \\ D. Helbig ${ }^{*}$; R. da S. Michaello ${ }^{2}$ E E. D. dos Santos ${ }^{2}$; M. de V. Real²; L. A. O. \\ Rocha $^{1}$; L. A. Isoldi ${ }^{2}$ \\ ${ }^{1}$ Programa de Pós-Graduação em Engenharia Mecânica (PROMEC), Universidade Federal do Rio Grande do Sul \\ (UFRGS), 90050-170, Porto Alegre-RS, Brasil \\ ${ }^{2}$ Programa de Pós-Graduação em Modelagem Computacional (PPGMC), Universidade Federal do Rio Grande \\ (FURG), 96203-900, Rio Grande-RS, Brasil \\ *daniel.helbig@gmail.com
}

(Recebido em 14 de setembro de 2014; aceito em 29 de dezembro de 2014)

\begin{abstract}
Placas finas de aço são elementos estruturais muito utilizados em diversas aplicações de engenharia, como em plataformas offshore, pontes, navios, aviões e automóveis. Porém, em algumas situações específicas as placas podem sofrer flambagem térmica, que é uma deflexão lateral causada simplesmente por um aumento de temperatura (variação crítica de temperatura). Este artigo utilizou um modelo computacional desenvolvido no software ANSYS, que é baseado no Método dos Elementos Finitos (MEF), para tratar o problema da flambagem térmica em placas. O modelo numérico foi verificado através da comparação de seus resultados com a solução analítica do problema. Foram analisadas placas com diferentes relações de $H / L$, sendo $H$ a altura e $L$ o comprimento da placa. Além disso, diferentes espessuras para a placa foram também consideradas. Os resultados indicam que o aumento da variação crítica de temperatura está relacionado tanto ao aumento da relação $H / L$ como também com o aumento da espessura da placa.
\end{abstract}

Palavras-chave: Flambagem térmica, placas finas de aço, Método dos Elementos Finitos (MEF).

Thin steel plates are structural elements widely used in several engineering applications, as in offshore platforms, bridges, ships, aircrafts and cars. However, in some specific situations the plates can suffer thermal buckling, which is a lateral deflection and bending caused only by a rise in temperature (critical temperature variation). This paper uses a computational model developed in ANSYS software, that is based on the Finite Element Method (FEM), to treat the thermal buckling problem in plates. The numerical model was verified by means of the comparison of its results with the analytical solution of the problem. Plates with different $H / L$ ratios, being $H$ the height and $L$ the length of the plate, were analyzed. Besides, different thickness of the plate were also considered. The results indicated that the increase in the critical thermal variation is related to the increase of the $H / L$ ratio as well as to the increase of the plate thickness.

Keywords: Thermal buckling, thin steel plates, Finite Element Method (FEM).

\section{INTRODUÇÃO}

Placas são elementos estruturais limitados por duas superfícies planas distanciadas entre si de uma grandeza designada por espessura. Quando a dimensão da espessura é muito menor do que as dimensões das superfícies planas limitantes, as placas são chamadas de placas finas. No caso de placas finas é possível estabelecer a chamada Teoria Clássica das Placas Finas [1], desenvolvida por Lagrange em 1811, para a qual são consideradas válidas as hipóteses de Kirchhoff.

Por serem elementos estruturais esbeltos, as placas são de uso constante em estruturas de edifícios, pontes, na indústria naval, aérea e automobilística. Muitos são os fatores que contribuem para a utilização de placas finas, dentre os quais pode-se citar a sensível diminuição do peso da estrutura. 
Sabe-se que elementos estruturais quando submetidos a tensões de tração podem sofrer deformação axial ou ruptura, entretanto quando estão sujeitos a tensões de compressão existe ainda uma terceira possibilidade: a flambagem. A flambagem é um fenômeno de instabilidade elástica que ocorre em componentes estruturais esbeltos e consiste na deflexão lateral do mesmo em relação à direção de atuação da tensão de compressão [2].

Na flambagem, no momento em que a carga crítica (ou tensão crítica) é atingida, ocorre uma súbita flexão do elemento estrutural em uma direção transversal a de aplicação do carregamento de compressão. Uma importante observação é que, em geral, a carga crítica (ou tensão crítica) que provoca a flambagem tem intensidade bastante inferior à carga (ou tensão) que causaria o escoamento do material do componente estrutural [3].

Normalmente a tensão de compressão em um elemento estrutural é causada por carregamento axial, momento fletor, força cortante, carga concentrada ou por combinação desses [3]. Entretanto existe ainda a possibilidade de um componente estrutural ser submetido a uma tensão de compressão devido a um aumento de temperatura, desde que o mesmo tenha o deslocamento de suas extremidades impedido pela presença de apoios. Nesse caso as tensões de compressão são chamadas de tensões térmicas [4].

Portanto quando o deslocamento axial de um elemento estrutural for restringido e esse componente for submetido a uma variação positiva (aumento) de temperatura, surgirá uma força interna de compressão e, consequentemente, uma tensão de compressão que, dependendo de sua magnitude, poderá causar uma flambagem térmica.

Não foram encontradas muitas publicações científicas sobre a flambagem térmica de placas. Em Ko [5] a flambagem mecânica e a flambagem térmica de placas retangulares com furos quadrados e circulares centralizados nas mesmas foram estudadas. Um modelo numérico baseado no MEF foi usado para estudar o efeito das condições de vinculação da placa, da relação de aspecto da placa e da geometria e tamanho dos furos da placa na flambagem mecânica e na flambagem térmica das mesmas. Entre outras conclusões, os resultados indicaram que o aumento do tamanho do furo conduz a um aumento na força necessária para a flambagem térmica. Já força de compressão para a flambagem mecânica pode também ser aumentada sob certas condições de vinculação e para algumas relações de aspecto, por exemplo, relação entre a altura e o comprimento. E em Murphy e Ferreira [6] a flambagem térmica de placas retangulares engastadas foi analisada. As análises desenvolvidas usaram a teoria de placas de von Karman para o estudo analítico da flambagem térmica. Os resultados analíticos foram comparados com resultados experimentais para a flambagem térmica e uma boa concordância entre os resultados foi obtida.

Nesse contexto, o presente trabalho tem como objetivo analisar numericamente a flambagem térmica em placas. Para isso um modelo computacional foi desenvolvido no software ANSYS, que é baseado no Método dos Elementos Finitos (MEF), para determinar a temperatura crítica de flambagem, ou seja, a temperatura na qual a placa sofre flambagem. Uma placa fina de aço engastada em dois bordos opostos e livre nos outros dois foi considerada. Várias relações entre a altura $(H)$ e o comprimento $(L)$ da placa com diferentes espessuras $(t)$ foram avaliadas, visando investigar a influência desses parâmetros na temperatura crítica de flambagem.

\section{MATERIAL E MÉTODOS}

Sabe-se que elementos estruturais, em diversas situações, são submetidos a variações de temperatura de pequena ou grande amplitude. A magnitude dessa variação de temperatura associada às propriedades específicas do material desse componente causará uma retração ou expansão do mesmo. A retração do componente acontece quando existe uma diminuição de temperatura, enquanto que a sua expansão acontece quando existe um aumento de temperatura. Se as condições de vinculação do elemento estrutural permitirem que a retração e a expansão aconteçam livremente, nenhuma tensão normal ocorrerá. Porém, se esse componente possuir condições de vinculação que impeçam sua deformação normal, surgirá uma tensão normal conhecida como tensão térmica. No caso da retração essa tensão será de tração, porém no caso 
da expansão a tensão será de compressão podendo causar flambagem térmica no componente estrutural.

Para determinar a temperatura que causa a flambagem térmica, ou seja, a temperatura crítica em placas foi empregado nesse trabalho um modelo computacional desenvolvido no software ANSYS. O software ANSYS é uma ferramenta computacional que usa como método de discretização o Método dos Elementos Finitos (MEF). Tal método permite análises estruturais lineares e não-lineares em problemas estáticos e dinâmicos [7].

Em linhas gerais, o MEF é baseado na divisão do domínio de integração, contínuo, em um número finito de pequenas regiões denominadas elementos finitos, transformando o meio contínuo em discreto. O comportamento de cada elemento é arbitrado de forma aproximada, com a condição de que a malha formada pelos elementos se comporte de forma semelhante ao contínuo original. No chamado modelo de deslocamentos do MEF, arbitra-se o campo de deslocamentos nodais e, como consequência, a interação de componentes de tensão entre elementos adjacentes é substituída pela interação de forças nodais entre elementos. Dessa maneira, o equilíbrio infinitesimal que se considera no modelo matemático de meio contínuo é substituído pelo equilíbrio de cada elemento finito isoladamente, trocando-se as equações diferenciais de equilíbrio por equações algébricas de equilíbrio do elemento como um todo. A partir destas equações algébricas escritas para cada elemento, obtém-se o sistema de equações de equilíbrio da malha de elementos. Esse sistema global, após a introdução das condições de vinculação ao meio exterior, permite a determinação da solução em termos dos deslocamentos nodais [8].

Para o modelo computacional usado no presente estudo foi adotado o elemento SHELL181, por ser um dos elementos do software ANSYS adequado para a simulação numérica de placas e cascas finas. O elemento SHELL181, apresentado na Figura 1, possui quatro nós e seis graus de liberdade por nó, ou seja, três translações nas direções $x, y$ e $z\left(u_{x}, u_{y}\right.$ e $\left.u_{z}\right)$ e três rotações em torno dos eixos $x, y$ e $z\left(r_{x}, r_{y}\right.$ e $\left.r_{z}\right)$, totalizando vinte e quatro graus de liberdade por elemento [7].

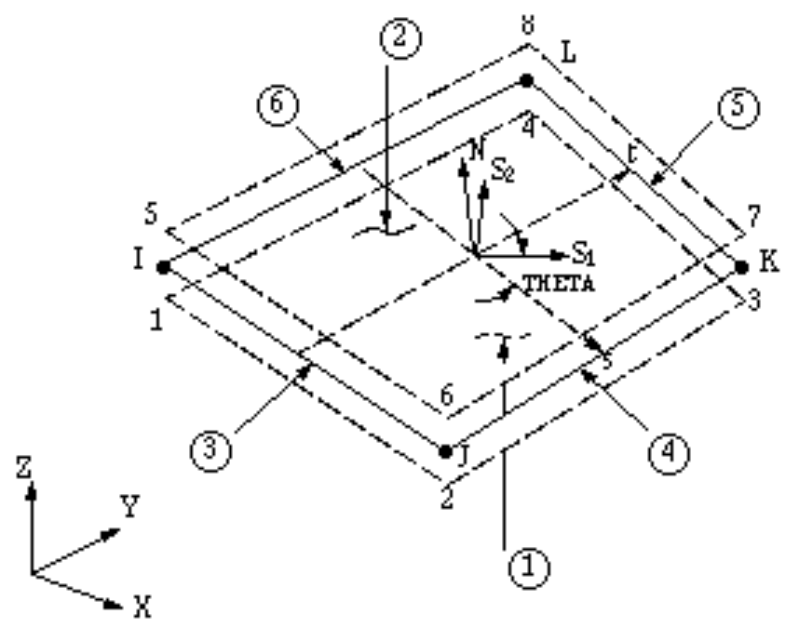

Figura 1: Elemento SHELL181.

Para o estudo numérico do fenômeno da flambagem térmica foi adotada a análise por autovalores. As equações de equilíbrio por elementos finitos para este tipo de análise envolvem a solução de equações algébricas homogêneas cujo autovalor mais baixo corresponde à temperatura crítica de flambagem e o autovetor associado representa o primeiro modo de flambagem. Essa solução é análoga à determinação da carga crítica de flambagem mecânica em uma placa, onde o autovalor mais baixo indica a carga crítica e o autovetor correspondente define o primeiro modo de flambagem [8]. Para isso a diferença entre a temperatura de referência e a temperatura aplicada na placa deve ser de $1{ }^{\circ} \mathrm{C}$, pois os autovalores e autovetores são calculados para uma carga unitária aplicada. Assim, com essa diferença de $1{ }^{\circ} \mathrm{C}$, o primeiro autovalor encontrado será igual à diferença de temperatura que causa a flambagem térmica [10]. 
No ANSYS o problema de autovalores-autovetores é resolvido usando o método numérico de Lanczos [7].

\subsection{Verificação do modelo computacional}

Para verificar o modelo computacional empregado nesse estudo foi considerada a solução analítica para uma placa retangular, simplesmente apoiada nos quatro lados e com movimentos no plano da placa impedidos em todo o contorno (Figura 2).



Figura 2: Placa simplesmente apoiada usada na verificação do modelo computacional.

A variação crítica de temperatura $\left(\Delta T_{c r}\right)$ analítica para a placa apresentada na Figura 2 é definida por [9]:

$$
\Delta T_{c r}=\min _{m, n}\left\{\frac{\pi^{2}\left[m^{2}\left(\frac{H}{L}\right)^{2}+n^{2}\right]}{12(1+v) \alpha\left(\frac{H}{t}\right)^{2}}\right\}
$$

onde: $m$ e $n$ são o número de meias ondas formadas na direções longitudinal e transversal da placa, respectivamente; $\pi$ é a constante matemática, $H$ é altura da placa $(\mathrm{mm}), L$ é o comprimento da placa $(\mathrm{mm})$; vé o coeficiente de Poisson do material da placa; $\alpha$ é o coeficiente de dilatação térmica $\left({ }^{\circ} \mathrm{C}^{-1}\right)$; e $t$ é a espessura da placa.

Aplicando a Equação (1) e considerando uma placa quadrada feita de aço com módulo de elasticidade $E=210000 \mathrm{MPa}$ e com $H=1000 \mathrm{~mm}, L=1000 \mathrm{~mm}, t=10 \mathrm{~mm}, v=0,3, \alpha=$ $12 \times 10^{-6}{ }^{\circ} \mathrm{C}^{-1}, m=1$ e $n=1$, a variação crítica de temperatura é $\Delta T_{c r}=10,54{ }^{\circ} \mathrm{C}$. Já a simulação numérica da flambagem térmica foi realizada empregando o modelo computacional proposto, onde essa mesma placa foi discretizada com elementos finitos quadrilaterais com tamanho característico de $20 \mathrm{~mm}$, obtendo um resultado para a variação crítica de temperatura de $\Delta T_{c r}=$ $10,50{ }^{\circ} \mathrm{C}$, existindo portanto uma diferença de $-0,38 \%$ em relação à solução analítica. A configuração do primeiro modo de flambagem da placa, formando uma meia onda nas direções longitudinal e transversal, pode ser observada na Figura 3. 


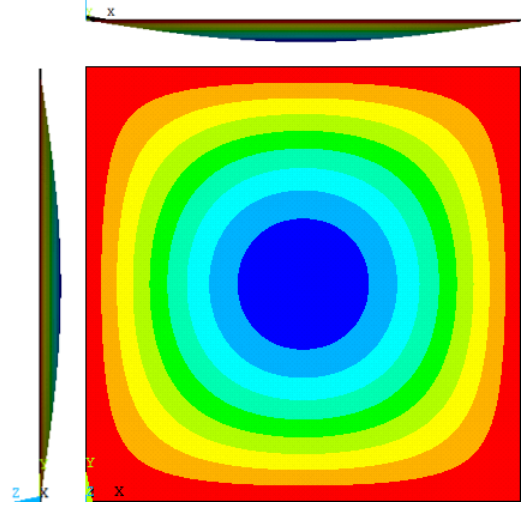

Figura 3: Configuração da placa quadrada devido à flambagem térmica.

Na sequência uma placa retangular com dimensões $H=1000 \mathrm{~mm}$ e $L=2000 \mathrm{~mm}$ e com os demais parâmetros idênticos ao da placa quadrada foi também considerada. Pela Equação (1) a variação crítica de temperatura encontrada foi de $\Delta T_{c r}=6,59{ }^{\circ} \mathrm{C}$ enquanto que através do modelo computacional um valor de $\Delta T_{c r}=6,58{ }^{\circ} \mathrm{C}$ foi obtido. Assim uma diferença de $-0,15 \%$ ocorreu entre o resultado numérico e o analítico. Na Figura 4 a configuração do primeiro modo para a flambagem térmica da placa retangular é apresentada, podendo ser observada a formação de apenas 1 meia onda em cada direção.

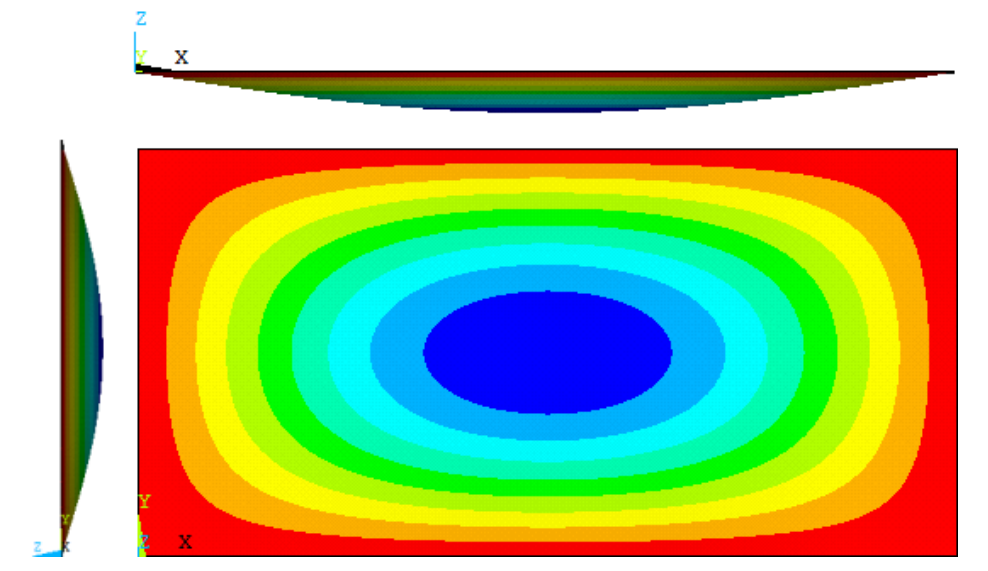

Figura 4: Configuração da placa retangular devido à flambagem térmica.

Levando em conta a excelente concordância entre os resultados numéricos gerados no modelo computacional desenvolvido no software ANSYS e os resultados analíticos definidos pela Equação (1) é possível afirmar que o modelo computacional foi devidamente verificado.

\section{RESULTADOS E DISCUSSÃO}

Como mencionado anteriormente, o objetivo deste artigo é avaliar numericamente a influência da relação $H / L$ em placas finas de aço, submetidas à flambagem térmica e com uma condição de vinculação diferente da considerada na verificação do modelo: engastada em dois bordos opostos e livre nos outros dois (Figura 5). Para isso o valor de $H$ será mantido fixo e igual a $1 \mathrm{~m}$, sendo empregadas as relações $H / L=0,40 ; 0,50 ; 0,67 ; 1,00 ; 1,25$ e 1,50 . O estudo envolve também, para todas as relações $H / L$, diferentes valores para a espessura da placa: $t=5$, 10,15 e $20 \mathrm{~mm}$, respectivamente. O domínio computacional, discretizado com uma malha regular de elementos finitos quadrilaterais (SHELL181), é esquematicamente representado na Figura 5. 


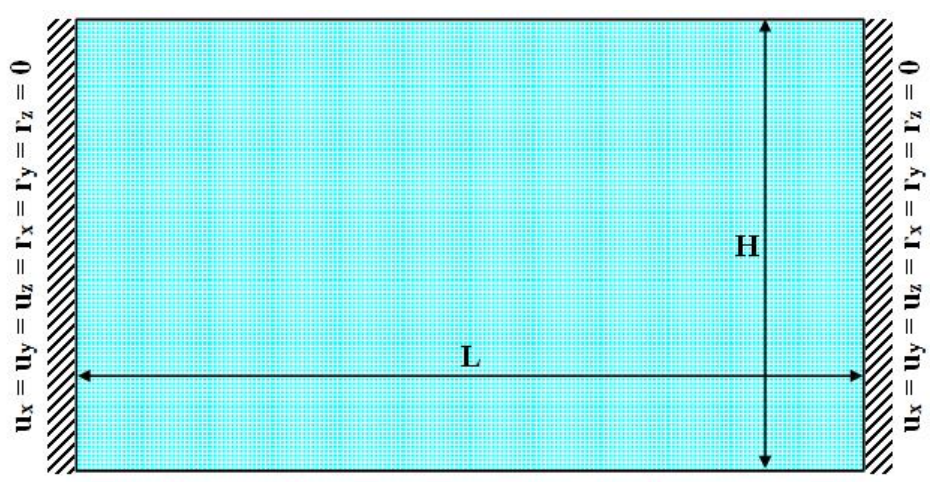

Figura 5: Domínio computacional do problema em estudo.

Um teste de independência de malha foi realizado, visando definir qual o refinamento de malha adequado para realizar as simulações numéricas. Na Figura 6 é apresentado o valor da variação crítica de temperatura em função do número de elementos finitos usados na discretização do domínio computacional.

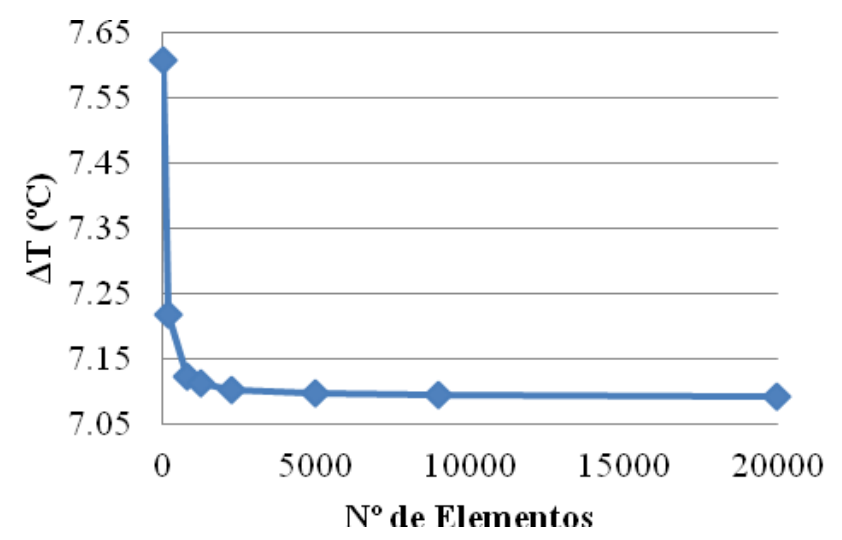

Figura 6: Relação entre o número de elementos e a variação da temperatura crítica.

Com base na Figura 6 é possível observar que a partir da malha gerada com 5000 elementos (com um tamanho característico do elemento de $20 \mathrm{~mm}$ ) não existe diferença significativa entre os valores encontrados para a variação crítica de temperatura, sendo, portanto, esse refinamento de malha considerado independente e adotado em todas as simulações numéricas realizadas.

Assim, na Figura 7 são apresentados os resultados obtidos para $\Delta T_{\text {cr }}$ de acordo com a variação de $H / L$ para as diferentes espessuras de placa consideradas.

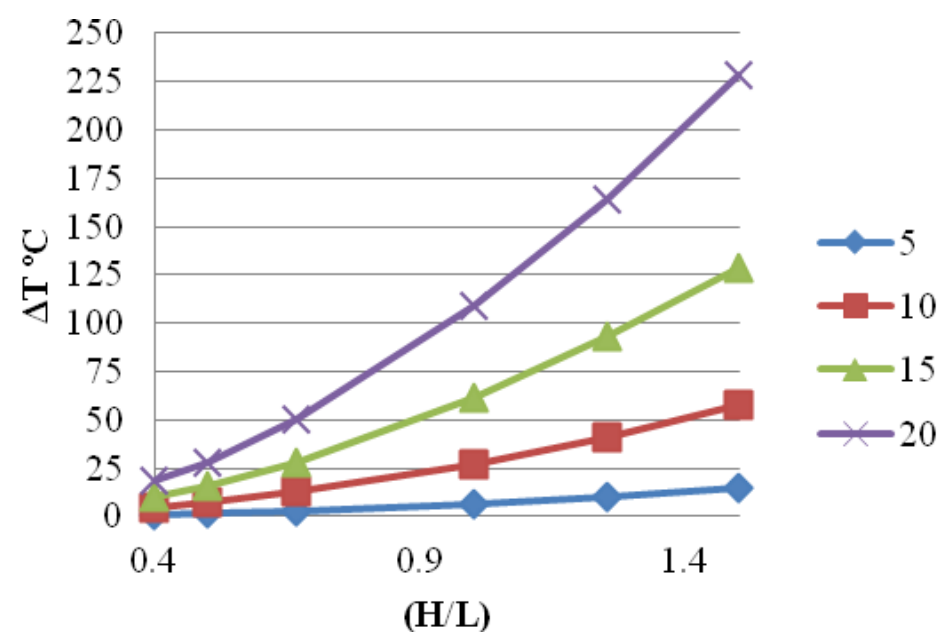

Figura 7: Variação da temperatura em função da relação $H / L$. 
Na Figura 7 é possível notar que existe uma mesma tendência no comportamento para todos os casos analisados. No que diz respeito à relação $H / L$, existe um aumento na variação crítica de temperatura à medida que $H / L$ aumenta. Esse comportamento já era esperado, pois as relações menores de $H / L$ são mais propícias à ocorrência da flambagem do que as que possuem uma alta relação $H / L$. Além disso, fica evidente que para maiores espessuras também são necessárias maiores variações de temperaturas para a ocorrência da flambagem.

Já a Figura 8 apresenta a $\Delta T_{\text {cr }}$ obtida por simulação numérica para as relações $H / L=0,40$ e 1,50 que são, respectivamente, as menores e maiores magnitudes para a variação crítica de temperatura entre os casos estudados. Sobre a relação $H / L=0,40$ encontram-se todas as menores $\Delta T_{\text {cr }}$, sendo que neste caso o menor valor encontrado refere-se a uma placa com espessura de $5 \mathrm{~mm}$, onde uma variação de apenas $1,14^{\circ} \mathrm{C}$ já causa flambagem térmica na placa, enquanto que o valor máximo para a variação crítica de temperatura foi de $\Delta T_{\mathrm{cr}}=18,21^{\circ} \mathrm{C}$ para a placa com $t=20 \mathrm{~mm}$. Já para a relação $H / L=1,50$ o mínimo valor encontrado de $\Delta T_{\text {cr }}$, que corresponde ao maior valor para uma placa de $5 \mathrm{~mm}$, foi de $14,41{ }^{\circ} \mathrm{C}$, enquanto o maior valor foi de $228,24^{\circ} \mathrm{C}$ para uma placa com espessura de $20 \mathrm{~mm}$.

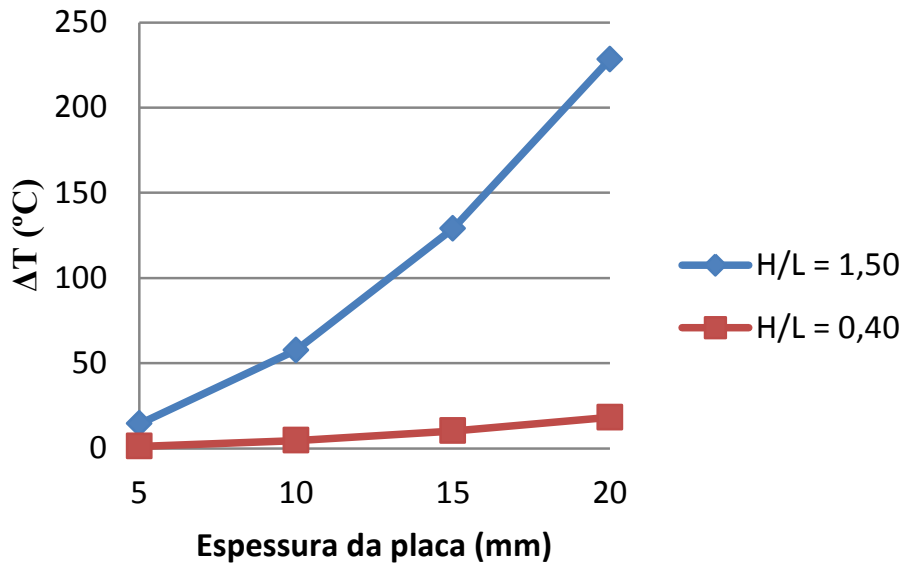

Figura 8: Variações críticas de temperatura para as relações $H / L=0,40$ e 1,50.

Por fim a Tabela 3 apresenta a diferença percentual entre as variações críticas de temperatura em relação à variação da espessura das placas. Observa-se que, dentro de uma faixa de $5 \mathrm{~mm}$ de espessura, a variação crítica de temperatura de uma placa de $5 \mathrm{~mm}$ para outra de $10 \mathrm{~mm}$ de espessura se mantém constante, cuja variação percentual é de aproximadamente $300 \%$. Quando se considera a variação de placas com espessura de $10 \mathrm{~mm}$ para placas com $15 \mathrm{~mm}$ este percentual, embora constante na faixa de $5 \mathrm{~mm}$, diminui para aproximadamente $125 \%$. Considerando-se ainda a situação em que a espessura da placa varia de $15 \mathrm{~mm}$ para $20 \mathrm{~mm}$, a variação da temperatura crítica diminui para aproximadamente $77 \%$. Em todas as situações analisadas não houve interferência da relação $H / L$, ou seja, o percentual de aumento da variação crítica de temperatura se manteve constante dentro da faixa considerada.

Tabela 3: Diferença percentual entre as variações críticas de temperatura em relação à espessura.

\begin{tabular}{lccc}
\hline \multirow{H}{*}{$\boldsymbol{H} / \boldsymbol{L}$} & \multicolumn{4}{c}{ \% $\boldsymbol{\Delta} \boldsymbol{T}_{\boldsymbol{c r}}$} \\
\cline { 2 - 4 } & $\boldsymbol{t}=\mathbf{5}$ e 10 $\mathbf{~ m m}$ & $\boldsymbol{t}=\mathbf{1 0}$ e $\mathbf{1 5} \mathbf{~ m m}$ & $\boldsymbol{t}=\mathbf{1 5}$ e $\mathbf{2 0} \mathbf{~ m m}$ \\
\hline 0,40 & 299,83 & 124,88 & 77,67 \\
\hline 0,50 & 299,80 & 124,85 & 77,63 \\
\hline 0,67 & 299,75 & 124,78 & 77,57 \\
\hline 1,00 & 299,57 & 125,10 & 77,38 \\
\hline 1,25 & 299,37 & 124,46 & 77,18 \\
\hline 1,50 & 299,16 & 124,23 & 76,93 \\
\hline
\end{tabular}


Os resultados apresentados na Tabela 3 indicam, como tendência, a diminuição gradativa da variação percentual da temperatura crítica em faixas de $5 \mathrm{~mm}$ conforme evidencia-se o aumento das espessuras das placas consideradas.

\section{CONCLUSÃO}

No presente trabalho foi analisado o problema da flambagem térmica para placas biengastadas. Para isso, foram consideradas placas com relações $H / L=1,50 ; 1,25 ; 1,00 ; 0,67$; 0,50 ; e 0,40 , e com espessuras $t=5 ; 10 ; 15$; e $20 \mathrm{~mm}$. Os resultados mostraram que a variação crítica de temperatura que provoca a flambagem cresce com o aumento da relação $H / L$ e com o aumento da espessura da placa.

Das configurações estudadas a que demonstrou, dentro de condições pré-estabelecidas, ser necessária a menor variação de temperatura para que se caracterize a flambagem térmica foi a relação $H / L=0,40$. E, em sentido contrário, àquela que necessitou de maior variação de temperatura para caracterizar a flambagem térmica foi à relação $H / L=1,50$.

Cabe destacar que este é um trabalho inicial dentro do grupo de pesquisa de Mecânica dos Sólidos Computacional da FURG sobre flambagem térmica, no qual ficou comprovada a eficácia do software ANSYS em modelar computacionalmente esse fenômeno físico. Portanto é justificada a continuidade desta pesquisa, avaliando a influência na flambagem térmica, por exemplo, de outras condições de contorno para as placas e também de perfurações nas mesmas. Estudos envolvendo a flambagem térmica em outros elementos estruturais, como as colunas, também podem ser desenvolvidos.

\section{AGRADECIMENTOS}

Ou autores agradecem à Universidade Federal de Rio Grande (FURG), à Universidade Federal de Rio Grande do Sul (UFRGS) e ao Conselho Nacional de Desenvolvimento Científico e Tecnológico (CNPq) pelo apoio.

\section{REFERÊNCIAS BIBLIOGRÁFICAS}

1. Timoshenko S, Krieger SW. Theory of plates and shells. New York: McGraw-Hill; 1959. 580 p.

2. Silva VD. Mechanics and strength of materials. New York: Springer; 2006. 529 p.

3. Akesson B. Plate buckling in bridges and other structure. New York: Taylor \& Francis; 2007. 163 p.

4. Hibbeler RC. Resistência dos Materiais. São Paulo: Pearson-Prentice Hall; 2010. 637 p.

5. Ko WL. Mechanical and thermal buckling behavior of rectangular plates with different central cutouts. Edwards. National Aeronautics and Space Administration (NASA); 1998.

6. Murphy KD, Ferreira D. Thermal buckling of rectangular plates. Int J Solids Struct. 2001 May;38(2223):3979-94, doi:10.1016/S0020-7683(00)00240-7.

7. ANSYS, User's Manual (version 10.0). Houston: Swanson Analysis System Inc; 2005.

8. Madenci E, Guven I. The finite element method and applications in engineering using ANSYS ${ }^{\circ}$. New York: Springer; 2006. $702 \mathrm{p}$.

9. Birman V. Plate Structures. New York: Springer; 2011.312 p.

10. Cassenti B. Thermal buckling of a 2D beam fixed at both ends. University of Connecticut; 2012. Disponível em: http://www.engr.uconn.edu/ cassenti/ 